\title{
Employment status at transplant influences ethnic disparities in outcomes after deceased donor kidney transplantation
}

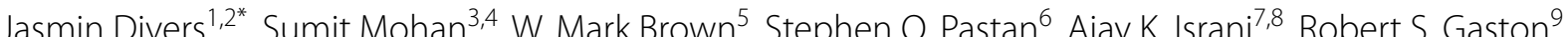
Robert Bray ${ }^{10}$, Shahidul Islam ${ }^{1,2}$, Natalia V. Sakhovskaya ${ }^{11}$, Alejandra M. Mena-Gutierrez ${ }^{11}$, Amber M. Reeves-Daniel ${ }^{11}$, Bruce A. Julian ${ }^{9}$ and Barry I. Freedman ${ }^{11}$

\begin{abstract}
Background: African American (AA) recipients of deceased-donor (DD) kidney transplants (KT) have shorter allograft survival than recipients of other ethnic groups. Reasons for this disparity encompass complex interactions between donors and recipients characteristics.

Methods: Outcomes from 3872 AA and 19,719 European American (EA) DDs who had one kidney transplanted in an AA recipient and one in an EA recipient were analyzed. Four donor/recipient pair groups (DRP) were studied, AA/AA, AA/EA, EA/AA, and EA/EA. Survival random forests and Cox proportional hazard models were fitted to rank and evaluate modifying effects of DRP on variables associated with allograft survival. These analyses sought to identify factors contributing to the observed disparities in transplant outcomes among AA and EA DDKT recipients.

Results: Transplant era, discharge serum creatinine, delayed graft function, and DRP were among the top predictors of allograft survival and mortality among DDKT recipients. Interaction effects between DRP with the kidney donor risk index and transplant era showed significant improvement in allograft survival over time in EA recipients. However, AA recipients appeared to have similar or poorer outcomes for DDKT performed after 2010 versus before 2001; allograft survival hazard ratios $(95 \% \mathrm{Cl})$ were $1.15(0.74,1.76)$ and $1.07(0.8,1.45)$ for AA/AA and EA/AA, compared to $0.62(0.54$, $0.71)$ and $0.5(0.41,0.62)$ for EA/EA and AA/EA DRP, respectively. Recipient mortality improved over time among all DRP, except unemployed AA/AAs. Relative to DDKT performed pre-2001, employed AA/AAs had HR $=0.37(0.2,0.69)$ versus $0.59(0.31,1.11)$ for unemployed AA/AA after 2010.

Conclusion: Relative to DDKT performed before 2001, similar or worse overall DCAS was observed among AA/AAs, while EA/EAs experienced considerable improvement regardless of employment status, KDRI, and EPTS. AA recipients of an AA DDKT, especially if unemployed, had worse allograft survival and mortality and did not appear to benefit from advances in care over the past 20 years.
\end{abstract}

Keywords: Deceased donor kidney transplantation, Allograft failure, Kidney recipient mortality, Outcome disparity, Employment status

*Correspondence: jasmin.divers@nyulangone.org

${ }^{1}$ Division of Health Services Research, Department of Foundations of Medicine, NYU Long Island School of Medicine, Mineola, NY 11501, USA

Full list of author information is available at the end of the article

\section{Background}

Deceased donor (DD) kidney transplantation (KT) from African American (AA) donors is associated with shorter allograft survival compared to DDKT from donors of other races/ethnicities. Donor African ancestry is 
included as a risk factor in the calculation of the Kidney Donor Risk Index (KDRI), a measure of DD organ quality used to generate the Kidney Donor Profile Index in the US kidney allocation system $[1,2]$. Similarly, AA recipients of DDKT have poorer outcomes, regardless of the race/ethnicity of the donor $[3,4]$.

Causes of ethnic differences in DDKT outcomes remain unclear; they are likely multifactorial, with inherited, environmental, and socioeconomic factors contributing to donor- and recipient-level effects. Several reports highlighted the adverse impact of genetics, poverty, geography, and lack of education on access to kidney transplantation and outcomes after engraftment [3,5-10]. We demonstrated more rapid allograft failure after kidney transplantation from DDs with apolipoprotein L1 gene (APOL1) high-risk genotypes. We suggested that using $A P O L 1$ genotyping instead of DD race might refine the KDRI by increasing the number of good quality kidneys for waitlisted recipients [11-15]. We and others reported genetic variants that affect AA DDKT outcomes either independently or through their interaction with APOL1 kidney-risk variants [16-19]. Beyond APOL1, several biological factors independently contribute to, or interact with non-biological factors leading to poorer outcomes among AA DDKT recipients. For example, given fewer AA donors and greater allelic variation at the HLA locus, potential AA recipients are disadvantaged in an allocation system that includes HLA matching. Despite recognizing these limitations and related changes, AA wait longer for kidney transplantation, an important modifiable risk factor for adverse outcomes [20-22]. The situation is compounded by complex interactions between donor and recipient characteristics impacting long-term outcomes.

Herein, we attempt to measure the effects of recipient- and donor-specific factors and their interaction on observed racial/ethnic disparities by studying partner kidneys from DDs that are, by definition, genetically identical and were transplanted into recipients of different races. Analyses were restricted to AA and European American (EA) donors and recipients for ease of comparison. This approach provides better control for donorlevel confounding factors, including donor-level genetic risk and race/ethnicity, on recipient outcomes after transplantation $[1,23]$.

\section{Methods}

These analyses used donor and recipient data in the Scientific Registry of Transplant Recipients (SRTR) for kidneys procured and transplanted between October 1, 1987, and June 30, 2016. Analyses were restricted to AA or EA DDs who had both partnered kidneys transplanted, one to an AA recipient and the other to an EA recipient, yielding four groups of donor/recipient pairs (DRP): AA/ AA, AA/EA, EA/AA, and EA/EA. This matched design better controlled for confounding by donor-related genetic, organ-specific, or socioeconomic factors and facilitated comparison of recipient-level factors contributing to observed racial disparities in outcomes. Donors or recipients $<18$ years of age were excluded.

The primary outcome was death-censored time to kidney allograft failure, determined by the interval between transplantation dates and allograft loss. In patients with a functioning allograft, the final observation date was censored for death with function or at last follow-up before March 5th, 2016. A secondary outcome treating death as a competing risk (CR) was also considered. In this case, the final observation date was censored at death for individuals who died with a functioning allograft or at the most recent follow-up before March 5th, 2016, for living individuals with functioning allografts.

A split-half hypothesis-free analysis approach was applied where a random survival forest (RSF) model was fit in a randomly selected subset of the data representing $50 \%$ of the data to rank variables and their interaction with DRP based on their variable importance (VIMP) measure [24, 25]. RSF models implementing the conditional VIMP measures are robust to multicollinearity between predictors and are well-suited to detect interaction effects, which are of particular importance here $[26,27]$. Analyses were repeated on the second half of the data and then on the complete data after observing strong reliability between the results obtained in the two subsets. Therefore, effect sizes and interaction effects with the DRP were estimated in the combined dataset using the top-ranked variables based on VIMP. This approach minimized the loss of statistical power caused by splitting the data into subsets [28]. Cox Proportional Hazard $(\mathrm{CPH})$ models were fitted for death-censored allograft survival (DCAS) and the Fine and Gray model when death was considered a CR to allograft survival to obtain effect size estimates. The sandwich estimator was used to obtain a robust estimation of the covariance matrix associated with the parameter estimates to account for the correlation between allograft failure rate and time to failure of kidneys donated by a single individual to two recipients. Lin and Wei reported that this sandwich estimator was consistent and robust to several misspecifications of the Cox model [29]. Proportional hazard assumptions were checked by visual inspection of the log-log curve and assessing the Schoenfeld and martingale residuals [30]. Models were fitted separately following missing data imputation, which was performed within the RSF framework because RSF based-imputations have demonstrated high degree of robustness even in the presence of non-random missingness patterns [31, 
Table 1 Demographic data for 23,591 deceased-donors (3872 African Americans and 19,719 European Americans)

\begin{tabular}{|c|c|c|c|c|c|c|c|}
\hline \multirow[t]{2}{*}{ Variable } & \multicolumn{2}{|l|}{ All } & \multicolumn{2}{|c|}{ AA donors } & \multicolumn{2}{|c|}{ EA donors } & \multirow[t]{2}{*}{$P$-value } \\
\hline & $N$ & Median (Q1, Q3), \% & $N$ & Median (Q1, Q3), \% & $N$ & Median (Q1, Q3), \% & \\
\hline Female, \% & 23,591 & 40.0 & 3872 & 35.4 & 19,719 & 40.9 & $<0.0001$ \\
\hline Age, years & 23,591 & $40.0(27.0,51.0)$ & 3872 & $35.0(24.0,47.0)$ & 19,719 & $41.0(28.0,51.0)$ & $<0.0001$ \\
\hline $\mathrm{BMI}, \mathrm{kg} / \mathrm{m}^{2}$ & 20,869 & $25.7(22.7,29.8)$ & 3529 & $25.8(22.8,30.1)$ & 17,340 & $25.7(22.7,29.8)$ & 0.05 \\
\hline Cardiac death, $\%$ & 19,499 & 9.7 & 3343 & 4.1 & 16,156 & 10.8 & $<0.0001$ \\
\hline $\mathrm{ECD}, \%$ & 23,591 & 14.0 & 3872 & 12.0 & 19,719 & 14.4 & $<0.0001$ \\
\hline Hypertension, \% & 23,591 & 21.4 & 3872 & 26.8 & 19,719 & 20.3 & $<0.0001$ \\
\hline Kidney Donor Risk Index (KDRI) & 19,395 & $1.3(1.1,1.7)$ & 3326 & $1.3(1.0,1.6)$ & 16,069 & $1.3(1.1,1.7)$ & $<0.0001$ \\
\hline Serum creatinine, mg/dL & 19,423 & $1.0(0.7,1.3)$ & 3329 & $1.1(0.9,1.5)$ & 16,094 & $0.9(0.7,1.2)$ & $<0.0001$ \\
\hline Cold ischemia time, hours & 22,327 & $16.0(11.0,22.9)$ & 3633 & $16.0(10.2,22.0)$ & 18,694 & $16.1(11.0,23.0)$ & $<0.0001$ \\
\hline Transplant era & & & & & & & $<0.0001$ \\
\hline Before 2001 & 23,591 & 37.8 & 3872 & 31.8 & 19,719 & 38.9 & \\
\hline $2001-2005$ & 23,591 & 17.6 & 3872 & 17.7 & 19,719 & 17.6 & \\
\hline $2005-2010$ & 23,591 & 21.3 & 3872 & 23.2 & 19,719 & 20.9 & \\
\hline After 2010 & 23,591 & 23.3 & 3872 & 27.2 & 19,719 & 22.6 & \\
\hline Diabetes, \% & 23,591 & 4.5 & 3872 & 5.2 & 19,719 & 4.3 & 0.01 \\
\hline $\mathrm{CMV}, \%$ & 23,561 & 59.3 & 3869 & 75.3 & 19,692 & 56.2 & $<0.0001$ \\
\hline $\mathrm{HCV}, \%$ & 19,474 & 2.7 & 3343 & 1.8 & 16,131 & 2.7 & 0.002 \\
\hline Alcohol use, \% & 23,591 & 18.0 & 3872 & 15.9 & 19,719 & 18.4 & 0.0001 \\
\hline Smoking, \% & 8773 & 67.8 & 1152 & 60.3 & 7621 & 68.9 & $<0.0001$ \\
\hline Cocaine use, $\%$ & 2444 & 44.4 & 444 & 59.7 & 2000 & 41.0 & $<0.0001$ \\
\hline Other drug, \% & 9298 & 43.5 & 1544 & 52.5 & 7754 & 41.7 & $<0.0001$ \\
\hline
\end{tabular}

Data presented as median $(\mathrm{Q} 1, \mathrm{Q} 3)$ for continuous variables and $\mathrm{N}(\%)$ for categorical variables

EA European American, AA African American, BMI Body mass index, ECD Extended-criteria donor, CMV Cytomegalovirus, HCV Hepatitis C virus antibody positive

32]. Ten imputed datasets were created, and the result obtained with these datasets were combined using established approaches [33-35]. Analyses were performed in SAS 9.4 and R 4.1. The RandomForestSCR package was used to fit Random Forest models for DCAS and the competing risk model [36].

\section{Results}

The cohort consisted of 47,182 kidney transplants from 3872 AA and 19,719 EA DDs. Tables 1 and 2 display distributions of demographic variables and clinical characteristics for donors and recipients, respectively. Data are presented as median $\left(\mathrm{Q}_{1}, \mathrm{Q}_{3}\right)$ for continuous and $\mathrm{N}(\%)$ for categorical variables. All comparisons in these Tables were statistically significant $(p<0.0001)$.

AA and EA DDs had comparable body mass index (BMI) and KDRI. Relative to EA DDs, AA DDs were more likely to be male (64.6\% vs. $59.1 \%$ ), younger (median age 35.0 vs. 40.9 years), cytomegalovirus (CMV) IgG antibody-positive $(75.3 \%$ vs. $56.2 \%)$, and diabetic $(5.2 \%$ vs. $4.3 \%)$. However, AA DDs were less likely to be smokers $(60.3 \%$ vs. $68.9 \%)$ or expanded-criteria donors (12\% vs. 14.4\%) (Table 1).
Independent of the race/ethnicity of the DD, AA recipients received their transplant at a younger age (median 48.0 vs. 51.0 years), were more likely to have been on dialysis (61.3\% vs. $50.9 \%)$, and had longer dialysis vintage ( 4.2 vs. 3.1 years). In addition, AA recipients were less likely to have received a prior transplant $(11.2 \%$ vs. $15.2 \%)$ ordie with a functioning allograft $(17.1 \%$ vs. $23.8 \%)$, but more likely to experience DGF (30.5\% vs.21.7\%) and had higher rates of acute rejection (1.8\% vs. $1.2 \%)$ (Table 2$)$. However, rates of immunosuppression medication use and the proportion of $\mathrm{KT}$ recipients needing induction therapy were comparable. Supplementary Table 1 show the demographics and clinical characteristics distribution by donor and recipient race.

Fig. 1 displays unadjusted death-censored allograft survival for KT recipients by DRP. Figure 1A shows the unadjusted allograft survival; differences in allograft survival outcomes are apparent between recipients based on race; the top two curves represent DCAS in EA recipients, and the bottom two curves display DCAS in AA recipients. Hazard ratios (HRs) (95\% CI) for EA/EA, AA/EA, and EA/AA DRPs, relative to AA/AA pairs, were $0.56(0.53,0.60), 0.65(0.59,0.70)$, and $0.96(0.91,1.02)$, respectively. Figure $1 \mathrm{~B}$ shows 
Table 2 Demographic and clinical characteristics of deceased-donor kidney transplant recipients

\begin{tabular}{|c|c|c|c|c|c|c|c|}
\hline \multirow[t]{2}{*}{ Variable } & \multicolumn{2}{|l|}{ All } & \multicolumn{2}{|l|}{$\mathrm{EA}$} & \multicolumn{2}{|l|}{$A A$} & \multirow[t]{2}{*}{$P$-value } \\
\hline & $\mathrm{N}$ & Median (Q1, Q3), \% & $\mathbf{N}$ & Median (Q1, Q3), \% & $\mathrm{N}$ & Median (Q1, Q3), \% & \\
\hline Female, \% & 47,182 & $38.10 \%$ & 23,591 & $37.10 \%$ & 23,591 & $39.00 \%$ & $<0.0001$ \\
\hline Age, years & 47,182 & $49.0(39.0,59.0)$ & 23,591 & $51(40.0,61.0)$ & 23,591 & $48(38.0,57.0)$ & $<0.0001$ \\
\hline $\mathrm{BMI}, \mathrm{kg} / \mathrm{m}^{2}$ & 40,139 & $26.8(23.3,31.1)$ & 20,211 & $26.3(23.0,30.4)$ & 19,928 & $27.3(23.7,31.6)$ & $<0.0001$ \\
\hline \multicolumn{8}{|l|}{ Education } \\
\hline High school or less, \% & 31,671 & $52.5 \%$ & 16,079 & $49.7 \%$ & 15,592 & $55.4 \%$ & $<0.0001$ \\
\hline Some college, $\%$ & 31,671 & $26.6 \%$ & 16,079 & $25.7 \%$ & 15,592 & $27.5 \%$ & $<0.0001$ \\
\hline College graduate, $\%$ & 31,671 & $20.8 \%$ & 16,079 & $24.5 \%$ & 15,592 & $17.0 \%$ & $<0.0001$ \\
\hline \multicolumn{8}{|l|}{ Primary insurance type } \\
\hline Medicaid, \% & 39,339 & $4.1 \%$ & 19,795 & $2.9 \%$ & 19,544 & $5.4 \%$ & $<0.0001$ \\
\hline Medicare, $\%$ & 39,339 & $65.8 \%$ & 19,795 & $60.4 \%$ & 19,544 & $71.3 \%$ & $<0.0001$ \\
\hline Private, $\%$ & 39,339 & $28.6 \%$ & 19,795 & $35.2 \%$ & 19,544 & $21.9 \%$ & $<0.0001$ \\
\hline Other, \% & 39,339 & $1.4 \%$ & 19,795 & $1.5 \%$ & 19,544 & $1.4 \%$ & $<0.0001$ \\
\hline Employed, \% & 41,308 & $44.4 \%$ & 20,709 & $47.6 \%$ & 20,599 & $41.2 \%$ & $<0.0001$ \\
\hline Graft duration, years & 47,182 & $4.1(1.6,7.8)$ & 23,591 & $4.5(1.8,8.3)$ & 23,591 & $3.9(1.5,7.2)$ & $<0.0001$ \\
\hline Early failure, \% & 47,182 & $7.30 \%$ & 23,591 & $6.50 \%$ & 23,591 & $8.00 \%$ & $<0.0001$ \\
\hline Graft failure, \% & 47,182 & $48.60 \%$ & 23,591 & $46.70 \%$ & 23,591 & $50.60 \%$ & $<0.0001$ \\
\hline Last Peak PRA, \% & 44,250 & $4.0(0.0,27.0)$ & 22,016 & $3.0(0.0,21.0)$ & 22,234 & $5.0(0.0,32.0)$ & $<0.0001$ \\
\hline Previous transplant, $\%$ & 46,989 & $13.2 \%$ & 23,492 & $15.2 \%$ & 23,497 & $11.2 \%$ & $<0.0001$ \\
\hline Last Peak PRA > 80\%, $\%$ & 44,250 & $10.4 \%$ & 22,016 & $9.4 \%$ & 22,234 & $11.4 \%$ & $<0.0001$ \\
\hline Previous kidney transplant, $\%$ & 46,989 & $11.9 \%$ & 23,492 & $13.1 \%$ & 23,497 & $10.7 \%$ & $<0.0001$ \\
\hline Previous dialysis, $\%$ & 47,182 & $56.1 \%$ & 23,591 & $50.9 \%$ & 23,591 & $61.3 \%$ & $<0.0001$ \\
\hline Time on dialysis, years & 21,318 & $3.7(2.2,5.6)$ & 9793 & $3.1(1.7,4.7)$ & 11,525 & $4.2(2.7,6.3)$ & $<0.0001$ \\
\hline Return to dialysis, $\%$ & 47,182 & $28.3 \%$ & 23,591 & $22.4 \%$ & 23,591 & $34.1 \%$ & $<0.0001$ \\
\hline Death with function, $\%$ & 47,182 & $20.4 \%$ & 23,591 & $23.8 \%$ & 23,591 & $17.1 \%$ & $<0.0001$ \\
\hline Death, \% & 47,182 & $43.6 \%$ & 23,591 & $45.0 \%$ & 23,591 & $42.1 \%$ & $<0.0001$ \\
\hline $\mathrm{DGF}, \%$ & 47,125 & $26.1 \%$ & 23,568 & $21.7 \%$ & 23,557 & $30.5 \%$ & $<0.0001$ \\
\hline Discharge serum creatinine, mg/dL & 45,784 & $2.3(1.5,4.5)$ & 22,932 & $2.0(1.3,3.7)$ & 22,852 & $2.6(1.6,5.3)$ & $<0.0001$ \\
\hline \multicolumn{8}{|l|}{ Cause of kidney failure } \\
\hline Type 1 diabetes, $\%$ & 37,099 & $5.9 \%$ & 18,717 & $8.0 \%$ & 18,382 & $3.8 \%$ & $<0.0001$ \\
\hline Type 2 diabetes, $\%$ & 37,099 & $15.1 \%$ & 18,717 & $13.6 \%$ & 18,382 & $16.7 \%$ & $<0.0001$ \\
\hline Polycystic kidney, \% & 47,182 & $6.0 \%$ & 23,591 & $9.5 \%$ & 23,591 & $2.5 \%$ & $<0.0001$ \\
\hline Glomerulonephritis, \% & 47,182 & $12.9 \%$ & 23,591 & $13.7 \%$ & 23,591 & $12.1 \%$ & $<0.0001$ \\
\hline Hypertension, \% & 47,182 & $21.4 \%$ & 23,591 & $11.7 \%$ & 23,591 & $31.0 \%$ & $<0.0001$ \\
\hline Induction therapy, \% & 47,182 & $75.5 \%$ & 23,591 & $75.9 \%$ & 23,591 & $75.1 \%$ & 0.05 \\
\hline Acute rejection, $\%$ & 47,182 & $1.5 \%$ & 23,591 & $1.2 \%$ & 23,591 & $1.8 \%$ & $<0.0001$ \\
\hline Lymphocyte-depleting, \% & 36,026 & $4.6 \%$ & 18,030 & $4.7 \%$ & 17,996 & $4.5 \%$ & 0.32 \\
\hline Immunosuppression, \% & 47,141 & $97.5 \%$ & 23,577 & $97.5 \%$ & 23,564 & $97.5 \%$ & 0.74 \\
\hline \multicolumn{8}{|l|}{ Immunosuppression class } \\
\hline Anti-proliferative, \% & 36,026 & $87.0 \%$ & 18,030 & $86.9 \%$ & 17,996 & $87.2 \%$ & 0.31 \\
\hline Calcineurin Inhibitor, \% & 36,026 & $96.6 \%$ & 18,030 & $96.6 \%$ & 17,996 & $96.6 \%$ & 0.68 \\
\hline mTOR Inhibitor, \% & 36,026 & $7.4 \%$ & 18,030 & $7.3 \%$ & 17,996 & $7.5 \%$ & 0.41 \\
\hline Corticosteroid, \% & 36,026 & $86.3 \%$ & 18,030 & $85.0 \%$ & 17,996 & $87.6 \%$ & $<0.0001$ \\
\hline EPTS & 38,657 & $1.6(1.0,2.1)$ & 19,673 & $1.6(1.1,2.1)$ & 18,984 & $1.5(1.0,2.0)$ & $<0.0001$ \\
\hline Other, $\%$ & 36,026 & $7.6 \%$ & 18,030 & $7.6 \%$ & 17,996 & $7.7 \%$ & 0.79 \\
\hline HCV-positive, $\%$ & 47,182 & $5.8 \%$ & 23,591 & $4.5 \%$ & 23,591 & $7.1 \%$ & $<0.0001$ \\
\hline Equivalent HLA mismatches (N) & 41,940 & $4.0(3.0,5.0)$ & 20,916 & $4.0(3.0,5.0)$ & 21,024 & $4.0(3.0,5.0)$ & $<0.0001$ \\
\hline
\end{tabular}

Data presented as median (Q1, Q3) for continuous variables and $\mathrm{N}(\%)$ for categorical variables

EA European American, AA African American, DGF Delayed graft failure, EPTS Estimated Post Transplant Survival, HCV Hepatitis C virus, HLA Human leukocyte antigen, mTOR Mammalian target of rapamycin, PRA Panel reactive antibody 

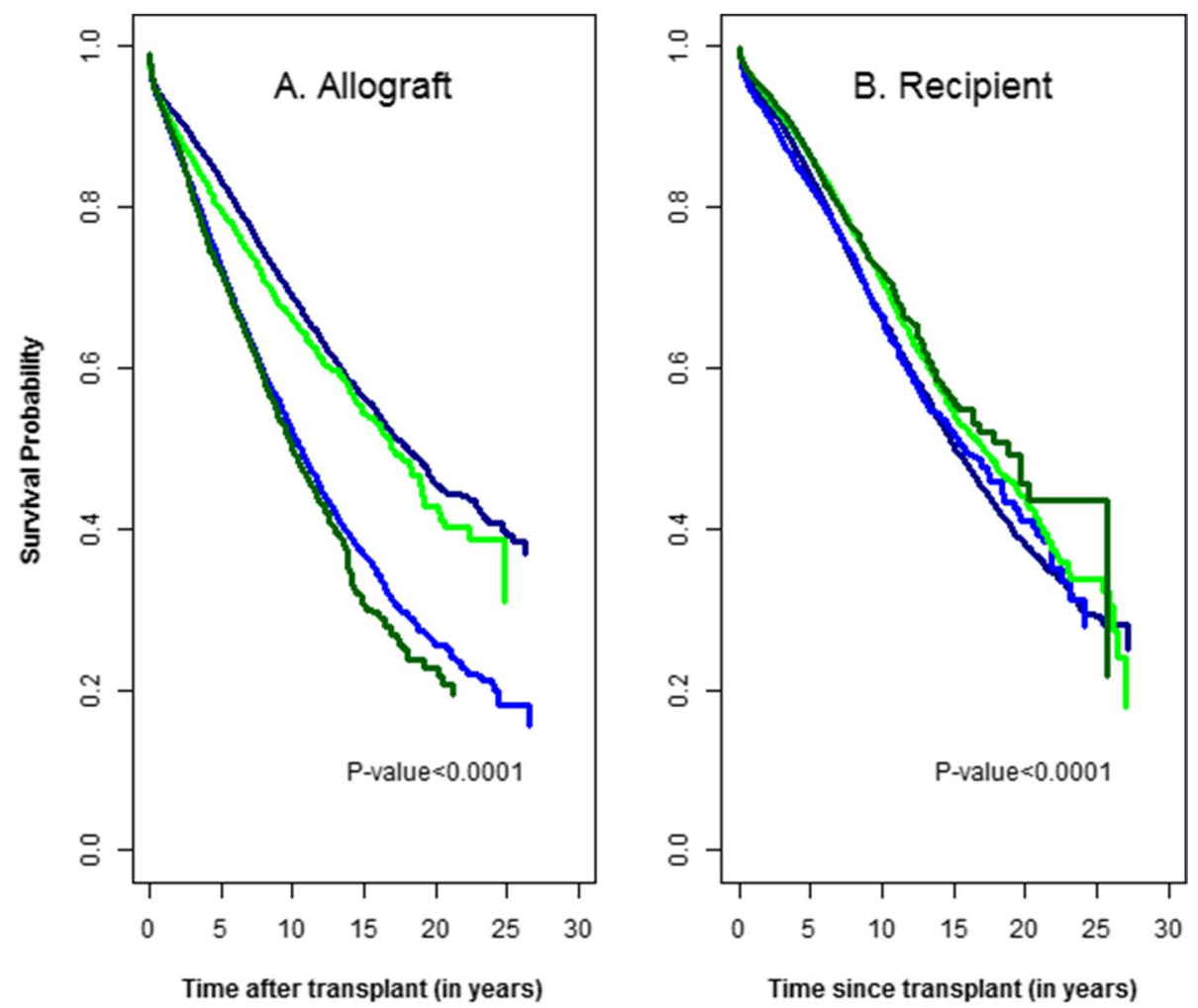

Fig. 1 Distribution of allograft survival by type of donor-recipient pair

EAVEA — EAVAA — AAVEA - AAVAA

unadjusted recipient survival, with mortality treated as a competing risk to allograft failure. At first glance, this graph suggests slightly higher recipient survival rates among AA/AA and EA/AA, compared to AA/EA and EA/EA DRP. However, it is important to keep in mind that AA recipients are approximately 3 years younger than EA recipients. Causes of graft failure did not vary between AA and EA recipients, except for the rate of non-compliance to immunosuppression medication, which was $11.9 \%$ among AA recipients, compared to 9.2\% for EA recipients.

The five-year DCAS rate improved among all four DRPs during the observation period (Supplementary Table 2). Five-year allograft survival rates in transplants performed after 2010 vs. before 2001 were $(0.74$ ( 0.52 , $0.90)$ vs. $0.64(0.60,0.67)$ for AA/AA DRPs, $0.85(0.76$, $0.94)$ vs. $0.74(0.71,0.77)$ for AA/EA, $0.83(0.81,0.86)$ vs. $0.64(0.63,0.65)$ for $\mathrm{EA} / \mathrm{AA}$, and $0.89(0.87,0.92)$ vs. $0.78(0.77,0.79)$ for EA/EA transplantations. Results of the random forest models, which inform the interaction tests that were subsequently performed can be found in Supplementary Table 3.
$\mathrm{CPH}$ models showed statistically significant interaction effects between the DRP with the transplant era (0.02), $\operatorname{KDRI}(p=0.0009)$, and EPTS $(p<0.0001)$ for DCAS.

The CR analysis helped clarify these results; it showed statistically significant interactions between the DRP and KDRI $(p<0.001)$ for allograft survival, and between the DRP with the KDRI $(p<0.0001)$, EPTS $(p=0.009)$, employment status $(p<0.0001)$ and transplant era $(p<0.0001)$ with kidney recipient mortality. Table 3 shows HRs for overall DCAS according to employment status and assuming no change in KDRI and EPTS. With employment EA/EA DRPs saw consistent improvement over time; for transplantations performed after 2010, HRs ranged from $0.42(0.37,0.47)$ to $0.46(0.41,0.51)$ for employed recipients and from $0.52(0.48,0.58)$ to 0.57 $(0.52,063)$ for unemployed recipients. Similar improvements were also observed with AA/EA pairs. However, for EA/AA DRPs, significant improvement in the overall DCAS was observed only post-2010 DDKTs, and the overall improvement was significantly smaller; HRs were $0.78(0.66,0.92)$ for EA/AA DRPs, compared to 0.42 $(0.38,0.47)$ for EA/EA's. 
Table 3 Hazard ratio and 95\% confidence interval (HR (95\% Cl)) for death-censored kidney allograft failure by DRP and transplant era, depending on employment status, and change in KDRI and EPTS score

\begin{tabular}{|c|c|c|c|c|c|c|c|c|c|}
\hline \multirow[t]{2}{*}{ DRP } & \multirow{2}{*}{$\begin{array}{l}\text { Transplant } \\
\text { era }\end{array}$} & \multicolumn{4}{|l|}{ Employed } & \multicolumn{4}{|c|}{ Unemployed } \\
\hline & & $\begin{array}{l}\mathrm{KDRI}=0, \\
\mathrm{EPTS}=0\end{array}$ & $\begin{array}{l}\mathrm{KDRI}=0, \\
\mathrm{EPTS}=0.25\end{array}$ & $\begin{array}{l}\mathrm{KDRI}=0.25 \\
\mathrm{EPTS}=0\end{array}$ & $\begin{array}{l}\mathrm{KDRI}=0.25, \\
\mathrm{EPTS}=0.25\end{array}$ & $\begin{array}{l}\mathrm{KDRI}=0, \\
\mathrm{EPTS}=0\end{array}$ & $\begin{array}{l}\mathrm{KDRI}=0, \\
\mathrm{EPTS}=0.25\end{array}$ & $\begin{array}{l}\mathrm{KDRI}=0.25, \\
\mathrm{EPTS}=0\end{array}$ & $\begin{array}{l}\mathrm{KDRI}=0.25 \\
\mathrm{EPTS}=0.25\end{array}$ \\
\hline AA/AA & 2001-2005 & $\begin{array}{l}1.26(0.97 \\
1.63)\end{array}$ & $\begin{array}{l}1.36(1.06 \\
1.73)\end{array}$ & $\begin{array}{l}1.17(0.91, \\
1.50)\end{array}$ & $\begin{array}{l}1.26(1.00 \\
1.60)\end{array}$ & $\begin{array}{l}1.43(1.10 \\
1.85)\end{array}$ & $\begin{array}{l}1.54(1.20 \\
1.97)\end{array}$ & $\begin{array}{l}1.46(1.14 \\
1.87)\end{array}$ & $1.57(1.25,1.98)$ \\
\hline AA/AA & 2005-2010 & $\begin{array}{l}1.08(0.82 \\
1.41)\end{array}$ & $\begin{array}{l}1.16(0.91 \\
1.50)\end{array}$ & $\begin{array}{l}1.00(0.77 \\
1.30)\end{array}$ & $\begin{array}{l}1.08(0.85 \\
1.38)\end{array}$ & $\begin{array}{l}1.22(0.94 \\
1.60)\end{array}$ & $\begin{array}{l}1.32(1.03 \\
1.70)\end{array}$ & $\begin{array}{l}1.25(0.97 \\
1.62)\end{array}$ & $1.35(1.06,1.72)$ \\
\hline AA/AA & After 2010 & $\begin{array}{l}0.93(0.70 \\
1.25)\end{array}$ & $\begin{array}{l}1.01(0.77 \\
1.32)\end{array}$ & $\begin{array}{l}0.87(0.66, \\
1.15)\end{array}$ & $\begin{array}{l}0.94(0.72 \\
1.22)\end{array}$ & $\begin{array}{l}1.06(0.80 \\
1.41)\end{array}$ & $\begin{array}{l}1.26(0.96 \\
1.65)\end{array}$ & $\begin{array}{l}1.08(0.82, \\
1.43)\end{array}$ & $1.17(0.90,1.52)$ \\
\hline AA/AA & Before 2001 & Reference & & & & & & & \\
\hline $\mathrm{AA} / \mathrm{EA}$ & $2001-2005$ & $\begin{array}{l}0.65(0.49, \\
0.85)\end{array}$ & $\begin{array}{l}0.72(0.56 \\
0.93)\end{array}$ & $\begin{array}{l}0.64(0.49 \\
0.83)\end{array}$ & $\begin{array}{l}0.71(0.56 \\
0.91)\end{array}$ & $\begin{array}{l}0.78(0.59 \\
1.03)\end{array}$ & $\begin{array}{l}0.87(0.67 \\
1.13)\end{array}$ & $\begin{array}{l}0.80(0.62 \\
1.03)\end{array}$ & $0.89(0.70,1.13)$ \\
\hline AA/EA & 2005-2010 & $\begin{array}{l}0.58(0.44 \\
0.76)\end{array}$ & $\begin{array}{l}0.65(0.50 \\
0.84)\end{array}$ & $\begin{array}{l}0.57(0.44 \\
0.75)\end{array}$ & $\begin{array}{l}0.64(0.50 \\
0.82)\end{array}$ & $\begin{array}{l}0.70(0.53 \\
0.93)\end{array}$ & $\begin{array}{l}0.78(0.60 \\
1.02)\end{array}$ & $\begin{array}{l}0.71(0.55 \\
0.93)\end{array}$ & $0.80(0.62,1.02)$ \\
\hline AA/EA & After 2010 & $\begin{array}{l}0.49(0.36 \\
0.66)\end{array}$ & $\begin{array}{l}0.54(0.41 \\
0.72)\end{array}$ & $\begin{array}{l}0.48(0.36 \\
0.64)\end{array}$ & $\begin{array}{l}0.54(0.41 \\
0.70)\end{array}$ & $\begin{array}{l}0.59(0.43 \\
0.80)\end{array}$ & $\begin{array}{l}0.68(0.51 \\
0.90)\end{array}$ & $\begin{array}{l}0.60(0.45 \\
0.80)\end{array}$ & $0.67(0.51,0.87)$ \\
\hline AA/EA & Before 2001 & Reference & & & & & & & \\
\hline EA/AA & $2001-2005$ & $\begin{array}{l}1.29(1.11 \\
1.49)\end{array}$ & $\begin{array}{l}1.36(1.18 \\
1.57)\end{array}$ & $\begin{array}{l}1.21(1.05 \\
1.40)\end{array}$ & $\begin{array}{l}1.28(1.11 \\
1.47)\end{array}$ & $\begin{array}{l}1.36(1.17 \\
1.58)\end{array}$ & $\begin{array}{l}1.44(1.24 \\
1.66)\end{array}$ & $\begin{array}{l}1.28(1.10 \\
1.48)\end{array}$ & $1.35(1.17,1.55)$ \\
\hline EA/AA & 2005-2010 & $\begin{array}{l}1.03(0.89 \\
1.20)\end{array}$ & $\begin{array}{l}1.09(0.94 \\
1.26)\end{array}$ & $\begin{array}{l}0.97(0.84, \\
1.12)\end{array}$ & $\begin{array}{l}1.02(0.89 \\
1.18)\end{array}$ & $\begin{array}{l}1.09(0.93 \\
1.27)\end{array}$ & $\begin{array}{l}1.15(0.99 \\
1.33)\end{array}$ & $\begin{array}{l}1.02(0.88, \\
1.19)\end{array}$ & $1.08(0.93,1.25)$ \\
\hline EA/AA & After 2010 & $\begin{array}{l}0.78(0.66 \\
0.92)\end{array}$ & $\begin{array}{l}0.82(0.70 \\
0.96)\end{array}$ & $\begin{array}{l}0.73(0.62 \\
0.86)\end{array}$ & $\begin{array}{l}0.77(0.66 \\
0.90)\end{array}$ & $\begin{array}{l}0.82(0.69 \\
0.97)\end{array}$ & $\begin{array}{l}0.87(0.74 \\
1.02)\end{array}$ & $\begin{array}{l}0.77(0.65 \\
0.91)\end{array}$ & $0.81(0.69,0.95)$ \\
\hline EA/AA & Before 2001 & Reference & & & & & & & \\
\hline EA/EA & $2001-2005$ & $\begin{array}{l}0.57(0.52, \\
0.62)\end{array}$ & $\begin{array}{l}0.62(0.57 \\
0.68)\end{array}$ & $\begin{array}{l}0.57(0.52 \\
0.62)\end{array}$ & $\begin{array}{l}0.62(0.57 \\
0.67)\end{array}$ & $\begin{array}{l}0.71(0.67 \\
0.76)\end{array}$ & $\begin{array}{l}0.78(0.73 \\
0.83)\end{array}$ & $\begin{array}{l}0.71(0.67 \\
0.75)\end{array}$ & $0.77(0.72,0.82)$ \\
\hline EA/EA & $2005-2010$ & $\begin{array}{l}0.52(0.48 \\
0.57)\end{array}$ & $\begin{array}{l}0.57(0.52 \\
0.62)\end{array}$ & $\begin{array}{l}0.52(0.48 \\
0.57)\end{array}$ & $\begin{array}{l}0.57(0.52 \\
0.62)\end{array}$ & $\begin{array}{l}0.65(0.61 \\
0.70)\end{array}$ & $\begin{array}{l}0.71(0.66 \\
0.76)\end{array}$ & $\begin{array}{l}0.65(0.61 \\
0.69)\end{array}$ & $0.71(0.66,0.75)$ \\
\hline EA/EA & After 2010 & $\begin{array}{l}0.42(0.38 \\
0.47)\end{array}$ & $\begin{array}{l}0.46(0.41 \\
0.51)\end{array}$ & $\begin{array}{l}0.42(0.38 \\
0.47)\end{array}$ & $\begin{array}{l}0.46(0.41 \\
0.51)\end{array}$ & $\begin{array}{l}0.53(0.48 \\
0.58)\end{array}$ & $\begin{array}{l}0.57(0.52 \\
0.63)\end{array}$ & $\begin{array}{l}0.52(0.48 \\
0.58)\end{array}$ & $0.57(0.52,0.63)$ \\
\hline EA/EA & Before 2001 & Reference & & & & & & & \\
\hline
\end{tabular}

Models were adjusted for recipient age at transplant, recipient sex, presence of DGF, previous dialysis, education level, recipient equivalent HLA mismatch, peak PRA recipient HCV status, cold ischemia time, donor age, donor CMV status, use of immunosuppressants, including use of lymphocyte depleting drugs, mTOR inhibitors and steroids

AA African American, EA European American

Table 4 shows HRs for the effect of DRP, KDRI, EPTS, and transplant era and employment status on recipient mortality with allograft failure as a CR. For transplantations performed before 2001 and assuming no change in KDRI and EPTS over time, reductions in mortality were observed among all four DRPs for employed DDKT. HRs for the post 2010 transplant era were 0.24 $(0.13,0.43), 0.27(0.17,0.45), 0.20(0.14,0.28), 0.24$ $(0.19,0.32)$ for AA/AA, AA/EA, EA/AA and AA/AA DRPs, respectively. In contrast, HRs for mortality were higher among unemployed recipients; $0.50(0.29,0.87)$, 0.55 (0.35, 0.87), $0.32(0.24,0.42)$, and $0.49(0.43,0.57)$ among these 4 DRPs, assuming no change in KDRI and EPTS. Figure 2 shows the disparity in recipient mortality according to employment status and DRP.

\section{Discussion}

Donor characteristics contribute to racial disparities in outcomes following DDKT $[2,23,37]$. The present study evaluated recipient factors potentially affecting ethnic disparities in DDKT outcomes using a unique donormatched design that controlled for genetic differences in transplanted kidneys, which allowed us to limit the impact of donor characteristics on DDKT outcomes, including many donor factors not available in the OPTN registry.

The analysis included 47,182 total kidney transplantations, 3872 involving AA DDs. As such, it is the most extensive analysis of its kind. Transplants resulting from the four possible DRPs had different DCAS, with EA recipients having better overall allograft survival than AA, independent from DD race/ethnicity. Analyses 
Table 4 Hazard ratio and 95\% confidence interval (HR (95\% CI)) for mortality as a competing risk to allograft failure by DRP and transplant era, depending on employment status, and change in KDRI and EPTS score

\begin{tabular}{|c|c|c|c|c|c|c|c|c|c|}
\hline \multirow[t]{2}{*}{ DRP } & \multirow{2}{*}{$\begin{array}{l}\text { Transplant } \\
\text { era }\end{array}$} & \multicolumn{4}{|l|}{ Employed } & \multicolumn{4}{|c|}{ Unemployed } \\
\hline & & $\begin{array}{l}\mathrm{KDRI}=0, \\
\mathrm{EPTS}=0\end{array}$ & $\begin{array}{l}\mathrm{KDRI}=0, \\
\mathrm{EPTS}=0.25\end{array}$ & $\begin{array}{l}\mathrm{KDRI}=0.25 \\
\mathrm{EPTS}=0\end{array}$ & $\begin{array}{l}\mathrm{KDRI}=0.25, \\
\mathrm{EPTS}=0.25\end{array}$ & $\begin{array}{l}\mathrm{KDRI}=0, \\
\mathrm{EPTS}=0\end{array}$ & $\begin{array}{l}\mathrm{KDRI}=0, \\
\mathrm{EPTS}=0.25\end{array}$ & $\begin{array}{l}\mathrm{KDRI}=0.25, \\
\mathrm{EPTS}=0\end{array}$ & $\begin{array}{l}\mathrm{KDRI}=0.25 \\
\mathrm{EPTS}=0.25\end{array}$ \\
\hline AA/AA & 2001-2005 & $\begin{array}{l}0.33(0.19 \\
0.55)\end{array}$ & $\begin{array}{l}0.36(0.22 \\
0.60)\end{array}$ & $\begin{array}{l}0.38(0.24 \\
0.63)\end{array}$ & $\begin{array}{l}0.43(0.27 \\
0.68)\end{array}$ & $\begin{array}{l}0.63(0.39 \\
1.02)\end{array}$ & $\begin{array}{l}0.70(0.44 \\
1.11)\end{array}$ & $\begin{array}{l}0.70(0.44 \\
1.11)\end{array}$ & $0.78(0.50,1.20)$ \\
\hline AA/AA & 2005-2010 & $\begin{array}{l}0.23(0.13 \\
0.39)\end{array}$ & $\begin{array}{l}0.25(0.15 \\
0.42)\end{array}$ & $\begin{array}{l}0.27(0.16 \\
0.44)\end{array}$ & $\begin{array}{l}0.30(0.18 \\
0.48)\end{array}$ & $\begin{array}{l}0.51(0.31 \\
0.85)\end{array}$ & $\begin{array}{l}0.57(0.35 \\
0.92)\end{array}$ & $\begin{array}{l}0.57(0.36 \\
0.92)\end{array}$ & $0.64(0.41,1)$ \\
\hline AA/AA & After 2010 & $\begin{array}{l}0.24(0.13 \\
0.43)\end{array}$ & $\begin{array}{l}0.27(0.15 \\
0.47)\end{array}$ & $0.28(0.16,0.5)$ & $\begin{array}{l}0.31(0.18 \\
0.54)\end{array}$ & $\begin{array}{l}0.50(0.29 \\
0.87)\end{array}$ & $\begin{array}{l}0.56(0.33 \\
0.95)\end{array}$ & $\begin{array}{l}0.56(0.33 \\
0.95)\end{array}$ & $0.63(0.38,1.03)$ \\
\hline AA/AA & Before 2001 & Reference & & & & & & & \\
\hline $\mathrm{AA} / \mathrm{EA}$ & $2001-2005$ & $\begin{array}{l}0.38(0.25 \\
0.60)\end{array}$ & $\begin{array}{l}0.43(0.28 \\
0.66)\end{array}$ & $\begin{array}{l}0.45(0.30 \\
0.69)\end{array}$ & $\begin{array}{l}0.51(0.34 \\
0.76)\end{array}$ & $\begin{array}{l}0.71(0.47 \\
1.06)\end{array}$ & $\begin{array}{l}0.79(0.53 \\
1.17)\end{array}$ & $\begin{array}{l}0.80(0.55 \\
1.18)\end{array}$ & $0.90(0.62,1.30)$ \\
\hline AA/EA & 2005-2010 & $\begin{array}{l}0.28(0.18 \\
0.45)\end{array}$ & $\begin{array}{l}0.32(0.20 \\
0.50)\end{array}$ & $\begin{array}{l}0.34(0.22 \\
0.52)\end{array}$ & $\begin{array}{l}0.38(0.25 \\
0.58)\end{array}$ & $\begin{array}{l}0.62(0.41 \\
0.94)\end{array}$ & $\begin{array}{l}0.69(0.46 \\
1.03)\end{array}$ & $\begin{array}{l}0.70(0.47 \\
1.04)\end{array}$ & $0.79(0.54,1.15)$ \\
\hline AA/EA & After 2010 & $\begin{array}{l}0.27(0.17 \\
0.45)\end{array}$ & $\begin{array}{l}0.31(0.19 \\
0.50)\end{array}$ & $\begin{array}{l}0.32(0.20 \\
0.52)\end{array}$ & $\begin{array}{l}0.36(0.23 \\
0.58)\end{array}$ & $\begin{array}{l}0.55(0.35 \\
0.87)\end{array}$ & $\begin{array}{l}0.61(0.39 \\
0.96)\end{array}$ & $\begin{array}{l}0.62(0.40, \\
0.96)\end{array}$ & $0.70(0.46,1.06)$ \\
\hline AA/EA & Before 2001 & Reference & & & & & & & \\
\hline EA/AA & $2001-2005$ & $\begin{array}{l}0.33(0.25 \\
0.45)\end{array}$ & $\begin{array}{l}0.35(0.26 \\
0.47)\end{array}$ & $\begin{array}{l}0.41(0.31 \\
0.55)\end{array}$ & $\begin{array}{l}0.43(0.33 \\
0.57)\end{array}$ & $\begin{array}{l}0.49(0.38 \\
0.63)\end{array}$ & $\begin{array}{l}0.52(0.40, \\
0.66)\end{array}$ & $\begin{array}{l}0.58(0.45 \\
0.74)\end{array}$ & $0.61(0.48,0.77)$ \\
\hline EA/AA & 2005-2010 & $\begin{array}{l}0.22(0.16 \\
0.31)\end{array}$ & $\begin{array}{l}0.24(0.17 \\
0.32)\end{array}$ & $0.28(0.2,0.37)$ & $\begin{array}{l}0.29(0.22 \\
0.39)\end{array}$ & $\begin{array}{l}0.39(0.29 \\
0.51)\end{array}$ & $\begin{array}{l}0.41(0.31 \\
0.53)\end{array}$ & $\begin{array}{l}0.45(0.35 \\
0.59)\end{array}$ & $0.48(0.37,0.62)$ \\
\hline EA/AA & After 2010 & $\begin{array}{l}0.20(0.14 \\
0.28)\end{array}$ & $0.21(0.15,0.3)$ & $\begin{array}{l}0.24(0.17 \\
0.34)\end{array}$ & $\begin{array}{l}0.26(0.18 \\
0.36)\end{array}$ & $\begin{array}{l}0.32(0.24 \\
0.42)\end{array}$ & $\begin{array}{l}0.33(0.25 \\
0.44)\end{array}$ & $\begin{array}{l}0.37(0.28 \\
0.49)\end{array}$ & $0.39(0.3,0.52)$ \\
\hline EA/AA & Before 2001 & Reference & & & & & & & \\
\hline EA/EA & $2001-2005$ & $\begin{array}{l}0.36(0.29 \\
0.44)\end{array}$ & $\begin{array}{l}0.40(0.32 \\
0.49)\end{array}$ & $\begin{array}{l}0.43(0.35 \\
0.52)\end{array}$ & $\begin{array}{l}0.47(0.39 \\
0.57)\end{array}$ & $\begin{array}{l}0.67 \text { (0.61, } \\
0.74)\end{array}$ & $\begin{array}{l}0.74(0.66, \\
0.82)\end{array}$ & $\begin{array}{l}0.76(0.69 \\
0.83)\end{array}$ & $0.84(0.76,0.92)$ \\
\hline EA/EA & $2005-2010$ & $\begin{array}{l}0.29(0.23 \\
0.36)\end{array}$ & $\begin{array}{l}0.32(0.26 \\
0.40)\end{array}$ & $\begin{array}{l}0.35(0.28 \\
0.43)\end{array}$ & $\begin{array}{l}0.38(0.31 \\
0.47)\end{array}$ & $\begin{array}{l}0.63(0.57 \\
0.71)\end{array}$ & $\begin{array}{l}0.70(0.63 \\
0.78)\end{array}$ & $\begin{array}{l}0.72(0.65 \\
0.80)\end{array}$ & $0.79(0.72,0.88)$ \\
\hline EA/EA & After 2010 & $\begin{array}{l}0.24(0.19 \\
0.32)\end{array}$ & $\begin{array}{l}0.27(0.21 \\
0.35)\end{array}$ & $\begin{array}{l}0.29(0.23 \\
0.38)\end{array}$ & $\begin{array}{l}0.32(0.25 \\
0.41)\end{array}$ & $\begin{array}{l}0.49 \text { (0.43 } \\
0.57)\end{array}$ & $\begin{array}{l}0.54(0.47 \\
0.63)\end{array}$ & $\begin{array}{l}0.56(0.49 \\
0.65)\end{array}$ & $0.62(0.53,0.71)$ \\
\hline EA/EA & Before 2001 & Reference & & & & & & & \\
\hline
\end{tabular}

Models were adjusted for recipient age at transplant, recipient sex, presence of DGF, previous dialysis, education level, recipient equivalent HLA mismatch, peak PRA recipient HCV status, cold ischemia time, donor age, donor CMV status, use of immunosuppressants, including use of lymphocyte depleting drugs, mTOR inhibitors and steroids

AA African American, EA European American

suggest that multiple factors contribute to kidney allograft outcomes. Some of the reported associations were described previously, including the well-known effects of DGF, serum creatinine at hospital discharge, recipient age, KDRI, EPTS, immunosuppressant medication, transplant era, donor age, etc. $[7,38,39]$ However, these effects are not modified by the DRP.

Employment status, KDRI, and EPTS interacted with DRP to affect DDKT outcomes. Unemployed recipients had worse DDKT allograft survival and mortality. Employment status was obtained before kidney transplantation. Recipients who reported working a full-time or a part-time job was considered employed; all others were considered unemployed, independently of the reason for not working. The HR estimates among unemployed recipients were almost twice those observed among employed recipients for mortality, although there was a minor overlap between confidence intervals in some cases.

Employment status at transplantation was the only socioeconomic variable that showed significant interaction effects with the DRP. The absence of independent effects of educational attainment and insurance status probably reflects the careful screening process of potential recipients by transplant programs. In contrast, employment status is rarely invoked as a reason to preclude active status of wait-listed transplant candidates in the US, despite its potential adverse effect on the ability to afford medications or access health insurance, especially after expiration of the 36-month post-transplant coverage provided by the Center for Medicare and Medicaid Services. The newly passed Immuno Bill indefinitely extends Medicare coverage of immunosuppressive drugs for KT recipients and may help reduce disparities in 

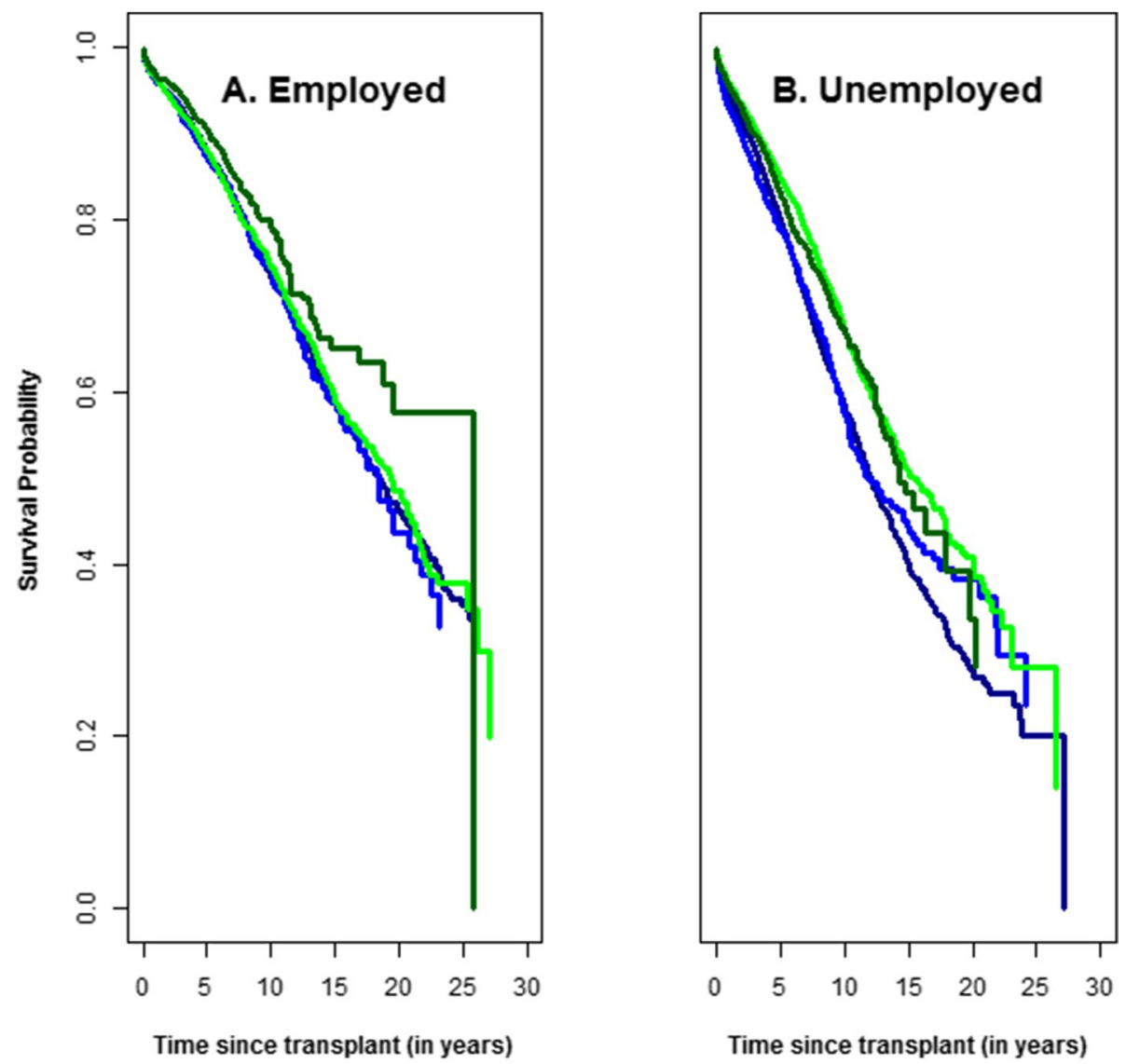

Fig. 2 Effect of employment on mortality by type of donor-recipient pair

long-term allograft survival. However, employment status may be a broader measure of social determinants of health with a clear association between unemployment, job loss, and retirement with poor outcomes.

In contrast, employment contributes to better physical health [40-42]. Unemployed individuals, independent of race/ethnicity, more often report feelings of depression and anxiety and high blood pressure, and tend to have higher rates of stroke, heart attack, and heart disease [43-45]. Unlike the composite scores considered in these analyses, employment status is a modifiable factor. Specific steps can be taken to understand how it affects outcomes among DDKT recipients and mitigate its effects.

Some measures reported in these analyses (e.g., KDRI and EPTS) are relatively new and were not previously part of the kidney allocation process. However, their utilization in these analyses ensures that comparisons across transplant eras are appropriate. KDRI includes donor race and other donor demographic and clinical characteristics. EPTS depends on recipient age, diabetes status, prior organ transplantations, and previous time on dialysis. Including these scores, the DRP, and the other variables in these models may have induced some collinearity. However, the random forests models are robust to multicollinearity. The KDRI score for AA donors is multiplied by a factor of 1.2, regardless of donor age, sex, and presence of other comorbidities. However, AA deceased donors were more likely to be younger and males such that the distributions of KDRI scores were comparable between AA and EA donors. The inclusion of these variables in the models was meant to help determine how socioeconomic and social determinants of health factors, which may interact with these scores, affect kidney transplant outcomes among AA and EA recipients.

Limitations of this report include potential underreporting in the SRTR database of various outcomes (e.g., DGF), mischaracterization of race and ethnicity, and viral infections, whose effects on KT outcomes were not initially recognized [46]. Analyses used registry data that were not collected for research purposes; therefore, 
some variables (e.g., employment status, medication use) may be incomplete and might not have been rigorously collected. However, it is unclear when the ongoing prospective APOL1 Long-term Kidney Transplantation Outcomes (APOLLO) study will accumulate enough events to address these questions [47]. These analyses provide some preliminary results that can be explored in other datasets.

Also, the study compared DDKT outcomes over more than 30 years, such that the standard of care and ways that measurements were collected and reported to the SRTR may have changed over time. However, focusing on four transplant eras should reduce these effects and their likelihood for confounding. These analyses were performed in a non-random subset of the SRTR data that may not have provided a representative sample of the distribution of outcomes observed among all DDKT recipients. For multiple reasons, including a greater need for kidney transplants in AA, lower rate of living kidney donation among AA, higher rates of HLA matching among individuals with recent African ancestry, waitlisted AA are more likely to receive AA DDKTs. Therefore, AA/AA DRP represents a significant proportion of all DDKTs [7, 48, 49].

\section{Conclusion}

AA recipients of kidney transplants from AA DDs had significantly shorter kidney allograft survival than EA recipients of AA DD kidneys and AA recipients of EA DD kidneys. Mortality among DDKT recipients remains high, especially among unemployed recipients, and does not appear to have changed since the early 2000s among unemployed AA recipients. Unemployment is associated with poorer outcomes among DDKT recipients, independent of race/ethnicity; however, its effects appeared to be consistently worse for AA DDKT recipients. Thus, improving outcomes for transplant recipients will require an improved understanding of the mechanisms by which socioeconomic factors, such as unemployment, adversely affect outcomes in the United States.

\footnotetext{
Abbreviations

AA: African American; APOL1: Apolipoprotein L1; APOLLO: APOL1 Long-term Kidney Transplantation Outcomes; BMl: Body mass index; BP: Blood pressure; CIT: cold ischemia time; CIT: Confidence interval; CKD: Chronic kidney disease; CMV: Cytomegalovirus; CPH: Cox proportional hazard model; CR: Competing risk; CVD: Cardiovascular disease; DCAS: Death-censored allograft survival; DD: Deceased donors; DDKT: Deceased donor kidney transplantation; DGF: Delayed graft function; DNA: Deoxyribonucleic acid; DRP: Donor/recipient pairs; EA: European American; EPTS: Estimated post-transplant survival; ESKD: End-stage kidney disease; HLA: Human Leucocyte antigen; HR: Hazard ratio; HRSA: Health Resources and Services Administration; KDRI: Kidney Donor Risk Index; KT: Kidney transplant / kidney transplantation; NUDT7: Nudix hydrolase 7 gene; OPTN: Organ Procurement and Transplantation Network; PRA: Panel reactive antibodies; RSF: Random survival forest; SEC63: Translocation protein SEC63 homolog; SNP: Single nucleotide polymorphism; SRTR: Scientific
}

Registry for Transplant Outcomes; T2D: Type 2 diabetes; UMOD: Uromodulin; UNOS: United Network for Organ Sharing; USRDS: United States Renal Data System; VIMP: Variable importance.

\section{Supplementary Information}

The online version contains supplementary material available at https://doi. org/10.1186/s12882-021-02631-4.

Additional file 1: Supplementary Table 1. Demographic and clinical characteristics by race/ethnicity of the donor-recipient pair. Supplementary Table 2. Five-year death-censored kidney allograft survival probability and 95\% confidence interval by DRP and transplant era. Supplementary Table 3. Predictor ranking based on variable importance for death-censored kidney allograft survival and allograft survival with mortality as a competing risk.

\section{Availability and of data and materials}

We do not have permission from SRTR to release the data used in these analyses. However, these data can be generated by obtaining access from SRTR and following the study design and analysis plan outlined in this manuscript.

\section{Disclaimer}

The data reported here have been supplied by the Hennepin Healthcare Research Institute (HHRI) as the Scientific Registry of Transplant Recipients (SRTR) contractor. Interpretation and reporting of these data are the responsibility of the author(s) and in no way should be seen as an official policy of or interpretation by the SRTR or the US Government.

\section{Authors' contributions}

Jasmin Divers designed the study, performed the statistical analyses, and drafted the manuscript. W. Mark Brown created the analysis dataset, contributed to the statistical analyses and interpretation of results. Sumit Mohan, Stephen Pastan, Ajay Israni, Robert S. Gaston, Robert Bray, Natalia V. Sakhovskaya, Alejandra M. Mena-Gutierrez, Amber M. Reeves-Daniel, and Bruce A. Julian contributed to the interpretation of results and preparation of the manuscript; Shahidul Islam contributed to the statistical analyses and interpretation of results; and Barry I Freedman designed the study, contributed in the statistical analyses, interpretation of results, and helped draft the manuscript. The author(s) read and approved the final manuscript.

\section{Funding}

This work was supported, in part, by grants from the National Institutes of Health R01 DK070941 (BIF), R01 DK084149 (BIF), R01 MD009055 (JD, BIF, BAJ), and Genomics of Transplantation U19-AI070119 (AKI, BAJ). The data reported here have been supplied by the Minneapolis Medical Research Foundation (MMRF) as the Scientific Registry of Transplant Recipients (SRTR) contractor. Interpretation and reporting of these data are the responsibilities of the authors, and in no way should be considered an official policy of, or interpretation by the SRTR or the United States Government. In addition, Dr. Mohan is supported by the National Institute of Diabetes and Digestive and Kidney Diseases (R01 DK1 14893 and U01116066) and the National Institute of Minority Health and Health Disparities (R01 MD014161).

\section{Declarations}

\section{Ethics approval and consent to participate}

This study used data from the SRTR that includes data on all donors, waitlisted candidates, and transplant recipients in the US, submitted by the Organ Procurement and Transplantation Network (OPTN) members. The Health Resources and Services Administration (HRSA) in the US Department of Health and Human Services provides oversight to the activities of the OPTN and SRTR contractors. Clinical and research activities are consistent with the Principles of the Declaration of Istanbul as outlined in the Declaration of Istanbul on Organ Trafficking and Transplant Tourism. 
The NYU Langone Institutional Review Board granted an exemption from requiring ethics approval on the ground that these analyses are conducted on de-identified data curated by the SRTR. Therefore, written consent was not required for this study based on the granted exemption.

\section{Consent for publication}

Not applicable.

\section{Competing interests}

Wake Forest University Health Sciences and Dr. Freedman have rights to an issued United States patent related to APOL1 genetic testing. In addition, Dr. Freedman receives research support from and is a consultant for AstraZeneca and RenalytixAl Pharmaceuticals. Dr. Mohan is a member of the Scientific Advisory Board for Angion Biomedica and is the deputy editor for Kidney International Reports. The other authors of this manuscript have no conflict of interest to disclose. Results presented in this paper have not been published previously in whole or part, except in abstract format.

\section{Author details}

'Division of Health Services Research, Department of Foundations of Medicine, NYU Long Island School of Medicine, Mineola, NY 11501, USA. ${ }^{2}$ Winthrop Research Institute, NYU Long Island School of Medicine, Mineola, NY, USA. ${ }^{3}$ Division of Nephrology, Department of Medicine, Columbia University College of Physicians \& Surgeons, New York, NY, USA. ${ }^{4}$ Department of Epidemiology, Mailman School of Public Health, Columbia University, NY, NY, USA. ${ }^{5}$ Department of Biostatistics and Data Sciences, Division of Public Health Sciences, Wake Forest School of Medicine, Winston-Salem, NC, USA. ${ }^{6}$ Renal Division, Department of Medicine, Emory University School of Medicine, Atlanta, GA, USA. ${ }^{7}$ Division of Nephrology, Department of Medicine, Hennepin Healthcare, University of Minnesota, Minneapolis, MN, USA. ${ }^{8}$ Minneapolis Medical Research Foundation, Minneapolis, MN, USA. ${ }^{9}$ University of Alabama at Birmingham School of Medicine, Birmingham, AL, USA. ${ }^{10}$ Department of Pathology \& Lab Medicine, Emory School of Medicine, Atlanta, GA, USA ${ }^{11}$ Department of Internal Medicine, Section on Nephrology, Wake Forest School of Medicine, Winston-Salem, NC, USA.

Received: 27 August 2021 Accepted: 1 November 2021

Published online: 03 January 2022

\section{References}

1. Callender CO, Cherikh WS, Traverso P, Hernandez A, Oyetunji T, Chang D. Effect of donor ethnicity on kidney survival in different recipient pairs: an analysis of the OPTN/UNOS database. Transplant Proc. 2009;41:4125-30.

2. Molnar MZ, Kovesdy CP, Bunnapradist S, Streja E, Krishnan M, Mucsi I, et al. Donor race and outcomes in kidney transplant recipients. Clin Transpl. 2013:27:37-51.

3. Kasiske BL, Neylan JF III, Riggio RR, Danovitch GM, Kahana L, Alexander SR, et al. The effect of race on access and outcome in transplantation. N Engl J Med. 1991:324:302-7.

4. Young CJ, Gaston RS. Renal transplantation in black Americans. N Engl J Med. 2000;343:1545-52

5. Mohan S, Mutell R, Patzer RE, Holt J, Cohen D, McClellan W. Kidney transplantation and the intensity of poverty in the contiguous United States. Transplantation. 2014;98:640-5.

6. Hall YN. Understanding racial differences in deceased-donor kidney transplantation: geography, poverty, language, and health insurance coverage. Dial Transplant. 2011:40:401-6.

7. Malek SK, Keys BJ, Kumar S, Milford E, Tullius SG. Racial and ethnic disparities in kidney transplantation. Transpl Int. 2011:24:419-24.

8. Axelrod DA, Dzebisashvili N, Schnitzler MA, Salvalaggio PR, Segev DL, Gentry SE, et al. The interplay of socioeconomic status, distance to center, and interdonor service area travel on kidney transplant access and outcomes. Clin J Am Soc Nephrol. 2010;5:2276-88.

9. Patzer RE, Amaral S, Wasse H, Volkova N, Kleinbaum D, McClellan WM. Neighborhood poverty and racial disparities in kidney transplant waitlisting. J Am Soc Nephrol. 2009;20:1333-40.

10. Patzer RE, Perryman JP, Schrager JD, Pastan S, Amaral S, Gazmararian JA, et al. The role of race and poverty on steps to kidney transplantation in the southeastern United States. Am J Transplant. 2012;12:358-68.
11. Bostrom MA, Kao WH, Li M, Abboud HE, Adler SG, lyengar SK, et al. Genetic association and gene-gene interaction analyses in African American dialysis patients with nondiabetic nephropathy. Am J Kidney Dis. 2012;59:210-21.

12. Freedman $B I$, Julian BA, Pastan $\mathrm{SO}$, Israni AK, Schladt D, Gautreaux MD, et al. Apolipoprotein L1 gene variants in deceased organ donors are associated with renal allograft failure. Am J Transplant. 2015;15:1615-22.

13. Freedman BI, Pastan SO, Israni AK, Schladt D, Julian BA, Gautreaux MD, et al. APOL1 genotype and kidney transplantation outcomes from deceased African American donors. Transplantation. 2016;100:194-202.

14. Reeves-Daniel AM, DePalma JA, Bleyer AJ, Rocco MV, Murea M, Adams PL, et al. The APOL1 gene and allograft survival after kidney transplantation. Am J Transplant. 2011;11:1025-30.

15. Julian BA, Gaston RS, Brown WM, Reeves-Daniel AM, Israni AK, Schladt DP, et al. Effect of replacing race with apolipoprotein L1 genotype in calculation of kidney donor risk index. Am J Transplant. 2017;17:1540-8.

16. Ma J, Divers J, Palmer ND, Julian BA, Israni AK, Schladt D, et al. Deceased donor multidrug resistance protein 1 and caveolin 1 gene variants may influence allograft survival in kidney transplantation. Kidney Int. 2015;88:584-92.

17. Divers J, Ma L, Brown WM, Palmer ND, Choi Y, Israni AK, et al. GWAS for time to failure of kidney transplants from African American deceased donors. Clin Transpl. 2020;34(6):e13827.

18. Moore J, McKnight AJ, Simmonds MJ, Courtney AE, Hanvesakul R, Brand OJ, et al. Association of Caveolin-1 gene polymorphism with kidney transplant fibrosis and allograft failure. JAMA. 2010;303:1282-7.

19. Cattaneo D, Ruggenenti P, Baldelli S, Motterlini N, Gotti E, Sandrini S, et al. ABCB1 genotypes predict cyclosporine-related adverse events and kidney allograft outcome. J Am Soc Nephrol. 2009;20:1404-15.

20. Roberts JP, Wolfe RA, Bragg-Gresham JL, Rush SH, Wynn JJ, Distant DA, et al. Effect of changing the priority for HLA matching on the rates and outcomes of kidney transplantation in minority groups. N Engl J Med. 2004:350:545-51.

21. Meier-Kriesche HU, Port FK, Ojo AO, Rudich SM, Hanson JA, Cibrik DM, et al. Effect of waiting time on renal transplant outcome. Kidney Int. 2000:58:1311-7.

22. Meier-Kriesche HU, Kaplan B. Waiting time on dialysis as the strongest modifiable risk factor for renal transplant outcomes: a paired donor kidney analysis. Transplantation. 2002;74:1377-81.

23. Locke JE, Warren DS, Dominici F, Cameron AM, Leffell MS, McRann $D A$, et al. Donor ethnicity influences outcomes following deceaseddonor kidney transplantation in black recipients. J Am Soc Nephrol. 2008;19:2011-9.

24. Ishwaran H, Kogalur UB, Gorodeski EZ, Minn AJ, Lauer MS. Highdimensional variable selection for survival data. J Am Stat Assoc. 2010;105:205-17.

25. Behnamian A, Millard K, Banks SN, White L, Richardson M, Pasher J. A systematic approach for variable selection with random forests: achieving stable variable importance values. IEEE Geosci Remote Sens Lett. 2017;14:1988-92.

26. van der Laan MJ. Statistical inference for variable importance. Int J Biostat. 2006;2:1-33.

27. Strobl C, Boulesteix A-L, Kneib T, Augustin T, Zeileis A. Conditional variable importance for random forests. BMC Bioinformatics. 2008;9:307.

28. Steyerberg EW, Uno H, loannidis JPA, van Calster B. Poor performance of clinical prediction models: the harm of commonly applied methods. J Clin Epidemiol. 2018:98:133-43.

29. Lin DY, Wei LJ. The robust inference for the cox proportional hazards model. J Am Stat Assoc. 1989;84:1074-8

30. Zhou B, Latouche A, Rocha $V$, Fine J. Competing risks regression for stratified data. Biometrics. 2011;67:661-70.

31. Tang F, Ishwaran H. Random Forest missing data algorithms. Stat Anal Data Min. 2017;10:363-77.

32. Kokla M, Virtanen J, Kolehmainen M, Paananen J, Hanhineva K. Random forest-based imputation outperforms other methods for imputing LC-MS metabolomics data: a comparative study. BMC Bioinformatics. 2019;20:492.

33. Little RJA, Rubin DB. Statistical analysis with missing data. 2nd ed. New York: John Wiley \& Sons; 2002.

34. Schafer JL. Analysis of incomplete multivariate data. New York: CRC Press; 1997. 
35. Li KH, Raghunathan TE, Rubin DB. Large-sample significance levels from multiply imputed data using moment-based statistics and an $\mathrm{F}$ reference distribution. J Am Stat Assoc. 1991;86:1065-73.

36. Ishwaran H, Gerds TA, Kogalur UB, Moore RD, Gange SJ, Lau BM. Random survival forests for competing risks. Biostatistics. 2014;15:757-73.

37. Cannon RM, Brock GN, Marvin MR, Slakey DP, Buell JF. The contribution of donor quality to differential graft survival in African American and Caucasian renal transplant recipients. Am J Transplant. 2012;12:1776-83.

38. Mannon RB. Delayed graft function: the AKI of kidney transplantation. Nephron. 2018;140:94-8.

39. Joshi S, Gaynor JJ, Bayers S, Guerra G, Eldefrawy A, Chediak Z, et al. Disparities among blacks, Hispanics, and whites in time from starting dialysis to kidney transplant waitlisting. Transplantation. 2013;95:309-18.

40. Hergenrather K, Zeglin R, McGuire-Kuletz M, Rhodes S. Employment as a social determinant of health: a systematic review of longitudinal studies exploring the relationship between employment status and physical health. Rehabil Res. 2015;29.

41. van der Noordt M, IJzelenberg H, Droomers M, Proper KI. Health effects of employment: a systematic review of prospective studies. Occup Environ Med. 2014;71:730-6.

42. Olesen SC, Butterworth P, Leach LS, Kelaher M, Pirkis J. Mental health affects future employment as job loss affects mental health: findings from a longitudinal population study. BMC Psychiatry. 2013;13:144.

43. Doede MS. Black jobs matter: racial inequalities in conditions of employment and subsequent health outcomes. Public Health Nurs. 2016:33:151-8.

44. Zagożdżon P, Parszuto J, Wrotkowska M, Dydjow-Bendek D. Effect of unemployment on cardiovascular risk factors and mental health. Occup Med (Lond). 2014;64:436-41.

45. Schultz WM, Kelli HM, Lisko JC, Varghese T, Shen J, Sandesara P, et al. Socioeconomic status and cardiovascular outcomes: challenges and interventions. Circulation. 2018;137:2166-78.

46. Israni AK, Li N, Cizman BB, Snyder J, Abrams J, Joffe M, et al. Association of donor inflammation- and apoptosis-related genotypes and delayed allograft function after kidney transplantation. Am J Kidney Dis. 2008:52:331-9.

47. Freedman BI, Moxey-Mims MM, Alexander AA, Astor BC, Birdwell KA, Bowden DW, et al. APOL1 long-term kidney transplantation outcomes network (APOLLO): design and rationale. Kidney Int Rep. 2020;5:278-88.

48. Lentine KL, Mandelbrot D. Addressing disparities in living donor kidney transplantation. Clin J Am Soc Nephrol. 2018;13:1909.

49. Rodrigue JR, Kazley AS, Mandelbrot DA, Hays R, LaPointe RD, Baliga P. Living donor kidney transplantation: overcoming disparities in live kidney donation in the US-recommendations from a consensus conference. Clin J Am Soc Nephrol. 2015;10:1687.

\section{Publisher's Note}

Springer Nature remains neutral with regard to jurisdictional claims in published maps and institutional affiliations.

Ready to submit your research? Choose BMC and benefit from:

- fast, convenient online submission

- thorough peer review by experienced researchers in your field

- rapid publication on acceptance

- support for research data, including large and complex data types

- gold Open Access which fosters wider collaboration and increased citations

- maximum visibility for your research: over $100 \mathrm{M}$ website views per year

At BMC, research is always in progress.

Learn more biomedcentral.com/submissions 